\title{
Soft mode and acoustic mode ferroelectric properties of deuterated triglycine sulphate crystal
}

\author{
ASHISH NAUTIYAL* and TRILOK CHANDRA UPADHYAY \\ Physics Department, H N B Garhwal University, Srinagar (Garhwal) 246 174, India
}

MS received 16 April 2012

\begin{abstract}
A mathematical study about deuterated triglycine sulphate $\left(\mathrm{CD}_{2} \mathrm{CD}_{2} \mathrm{COOD}\right)_{3} \mathrm{D}_{2} \mathrm{SO}_{4}$ crystal by a theoretical model which is extended with two sublattice pseudospin lattice coupled mode model by adding third, fourth and fifth order phonon anharmonic interaction terms as well as external electric field term in the crystal Hamiltonian. Double-time temperature dependent Green's function is used to derive soft mode frequency, dielectric permittivity, microwave absorption, quality factor, acoustic attenuation, electric conductivity, smooth function, relaxation time, ratio of figure of merits and respective applications in modern technologies. All theoretical results have a good agreement with experimental data.
\end{abstract}

Keywords. Pseudo spin-phonon interaction; anharmonic interactions; Green's function.

\section{Introduction}

Ferroelectric materials are novel materials, widely used in modern technology. They have a great potential of applications, in memory devices, infrared and pyroelectric detectors, transducers, display devices and piezoelectric devices. Triglycine group $\left(\mathrm{CH}_{2} \mathrm{NH}_{2} \mathrm{COOH}\right)_{3}[-\mathrm{X}-]_{2}[-\mathrm{Y}-]_{4}$ crystals are known as best pyroelectric materials in condensed matter physics. At large scale, each crystal of the group can be easily grown from water solution. All crystals are uniaxial ferroelectric crystals with Curie temperature ranging from 22 to $61^{\circ} \mathrm{C}$ or $295-334 \mathrm{~K}$ (table 1). All crystals in the group are monoclinic in both polar and non-polar phases with $2 / m$ and 2 space group points. All crystals show a second order phase transformation mechanism at different temperatures except deuterated triglycine selenate (Ist order) crystal. After transition, in each crystal mirror plane disappears and belongs to an isostructural transition. All triglycine group crystals have same key mechanism which is responsible for ferroelectric properties, i.e. three different possible set up of glycine ion (glycine I (GI), glycine II (GII) and glycine III (GIII)). Among these three different ions, GI has a coplanar configuration while GII and GIII have quasi-planar with mirror symmetry. This mirror symmetry tends to a double potential well system in the crystal which has trapped a hydrogen bond in its either well (figure 1). Quantum tunneling proposed a pseudo spin character of proton (hydrogen bond) between the wells to solve this problem. Pseudo spin character makes these crystals order and disorder type. That is why same model can be applied to every triglycine group crystal. In same series, Chaudhuri et al (1988) proposed a structural study of triglycine group crystals. Then,

*Author for correspondence (ashishnautiyal05@ rediffmail.com) phase transition in triglycine group crystals was initiated by Gonzalo (1970) who has used Ising spin model. Later, Tello and Hernandez (1973) applied molecular field approximation in tunneling model for same crystal system. And Blinc et al (1961) applied pseudospin model to triglycine group crystal. Much later, Chaudhuri et al (1988) applied Mitsui's (1958) model (which was proposed initially for hydrogen bonded order-disorder crystal, Rochelle Salt)), i.e. two sublattice pseudospin lattice coupled mode model.

\subsection{Deuterated triglycine sulphate (DTGS) crystal}

It is a deuterated form of triglycine sulphate crystal (Mathias et al 1956). DTGS is a successful high sensitive detector type material used as infrared spectrometer which takes measurements in the mid-infrared (mid IR) range. Crystal growth and its characteristics with doped condition were studied by Batra et al (2003). Domain structure of DTGS crystal has been investigated by Drozhdin et al (2006). Bjorkstam (1967) have shown a phase transition in deuterated triglycine sulphate crystal by deuteron nuclearmagnetic-resonance method. Ferroelectric properties of different solid solutions of DTGS crystal with different concentrations have been obtained by Brezina and Smutny (1968). Optical properties of deuterated triglycine sulphate have been investigated by Kushnir (2003). Dielectric properties of DTGS were proposed by Camnasio and Gonzalo (1975).

\section{Transformation of physical model into mathematical form}

\subsection{Model Hamiltonian}

For duterated triglycine sulphate crystal, we have extended two-sublattice pseudospin-lattice coupled mode model 
Table 1. Different crystal constants used in study (Chaudhuri et al 1988).

\begin{tabular}{|c|c|c|c|c|c|c|c|c|c|}
\hline $\begin{array}{l}\text { Ferroelectric } \\
\text { crystal name }\end{array}$ & Crystal class & $T_{\mathrm{c}}(\mathrm{K})$ & $C(\mathrm{~K})$ & $\begin{array}{c}\Omega \\
\left(\mathrm{cm}^{-1}\right)\end{array}$ & $\begin{array}{c}J \\
\left(\mathrm{~cm}^{-1}\right)\end{array}$ & $\begin{array}{c}V \\
\left(\mathrm{~cm}^{-1}\right)\end{array}$ & $\begin{array}{c}\hbar \omega \\
\left(\mathrm{cm}^{-1}\right)\end{array}$ & $\begin{array}{c}A \\
\left(\mathrm{~cm}^{-1}\right)\end{array}$ & $\begin{array}{c}N \mu \\
\left(\mu \mathrm{C} / \mathrm{cm}^{2}\right)\end{array}$ \\
\hline $\begin{array}{l}\text { Deuterated triglycine } \\
\text { sulphate }^{9} \text { (DTGS) }\end{array}$ & $\begin{array}{l}\text { Monoclinic with } 2 \mathrm{~m} \text {, } \\
2 \text { with space group }\end{array}$ & 333.86 & $4873 \cdot 16$ & $0 \cdot 01$ & 470 & 15 & 1.92 & $11 \cdot 15$ & $\begin{array}{c}2 \cdot 300 \\
\text { (at } 30 \cdot 70^{\circ} \mathrm{C} \text { ) }\end{array}$ \\
\hline
\end{tabular}

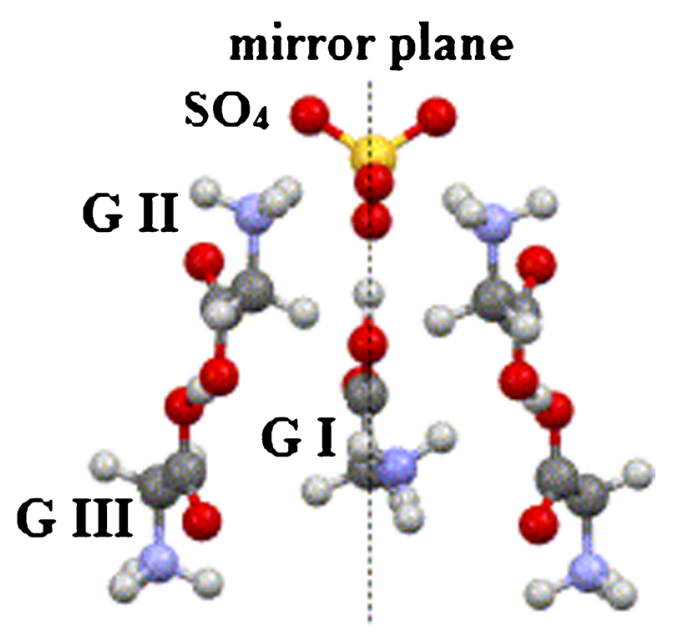

Figure 1. Crystal dynamics of every hydrogen bonded triglycine group crystals.

(Mitsui 1958) by adding third, fourth and fifth order phonon anharmonic interaction terms as well as external electric field term which is expressed as

$$
\begin{aligned}
! H= & -2 \Omega \sum_{i}\left(S_{1 i}^{x}+S_{2 i}^{x}\right)-\sum_{i j} J_{i j}\left[\left(S_{1 i}^{z} S_{2 i}^{z}\right)+\left(S_{2 i}^{z} S_{2 i}^{z}\right)\right] \\
& -\sum_{i j} K_{i j}\left(S_{1 i}^{z} S_{2 i}^{z}\right)-2 \mu E \sum_{i}\left(S_{1 i}^{z}+S_{2 i}^{z}\right) \\
& +\frac{1}{4} \sum_{k} \omega_{k}\left(A_{k} A_{k}^{+}+B_{k} B_{k}^{+}\right)-\sum_{i k} V_{i k} S_{1 i}^{z} A_{k} \\
& -\sum_{i k} V_{i k} S_{2 i}^{z} A_{k}^{+} \sum_{k_{1} k_{2} k_{3}} V^{(3)}\left(k_{1}, k_{2}, k_{3}\right) A_{k_{1}} A_{k_{2}} A_{k_{3}} \\
& +\sum_{k_{1} k_{2} k_{3} k_{4}} V^{(4)}\left(k_{1}, k_{2}, k_{3}, k_{4}\right) A_{k_{1}} A_{k_{2}} A_{k_{3}} A_{k_{4}}+\ldots \ldots,
\end{aligned}
$$

where, $\Omega$ is proton tunneling frequency, $S^{z}$ and $S^{x}$ are components of pseudospin variable, $J_{i j}$ the interaction between same lattices and $K_{i j}$ the interaction between different lattices. $\mu$ is dipole moment of $\mathrm{O}-\mathrm{H}-\mathrm{O}$ bond, $E$ the external electric field, $V$ the spin lattice interaction and $A_{k}$ and $B_{k}$ are position and momentum operators, $\omega_{k}$ the harmonic phonon frequency, and $V^{(3)}$ and $V^{(4)}$ are third and fourth order atomic force constants Drozhdin et al (2006).
Equation during calculation are solved based on basic physical rule: The particle of mass, $m$, is considered to move along $x$-axis with a wave function, $\Psi(x, t)$, which is solved by the Schrodinger wave equation:

$$
\frac{\hbar^{2}}{2 m} \frac{\partial^{2} \psi}{\partial x}+i \hbar \frac{\partial \psi}{\partial t}=V \psi
$$

This equation is equivalent to $F=m a$ (II ${ }^{\text {nd }}$ law Newton) in the above equation $\Psi(x, t)$ is a wavefunction at position, $x$ and time $t$. Born statistical gives the value of $|\Psi(x, t)|^{2}$ which also gives probability of finding the particle at $x$ position and time $t$ by the equation

$$
\tan k_{1 a}=-\frac{k_{1}}{k_{2}}=-\sqrt{\frac{E-V_{0}}{-E}}
$$

\subsection{Green's function, width, shift and soft mode frequency}

We consider the Green's function

$$
\begin{aligned}
G_{i j}\left(t-t^{\prime}\right) & =\left\langle\left\langle S_{1 i}^{z}(t) ; S_{1 j}^{z}\left(t^{\prime}\right)\right\rangle\right\rangle \\
& =-i \theta\left(t-t^{\prime}\right)\left\langle\left[S_{1 i}^{z}(t) ; S_{1 j}^{z}\left(t^{\prime}\right)\right]\right\rangle,
\end{aligned}
$$

in which $\theta\left(t-t^{\prime}\right)$ step function is zero for $t<t^{\prime}$ and unity for $t>t^{\prime}$. The angular bracket $<\ldots . .>$ denotes ensemble average over a grand canonical ensemble.

Spontaneous polarization in DTGS crystal is

$$
P_{\mathrm{s}}=2 N_{\mu}<s^{\mathrm{z}}>\text {. }
$$

In two dimensional form of pseudospin in z-directional, sum is not zero before $T_{\mathrm{C}}$ (Curie temperature).

$$
S_{1}^{\mathrm{Z}}+S_{1}^{\mathrm{Z}} \neq 0
$$

And $S_{1}^{\mathrm{Z}} \neq S_{1}^{\mathrm{Z}} ; \quad$ for $T<T_{\mathrm{C}}$.

But, after Curie temperature, total spontaneous polarization becomes zero.

$$
S_{1}^{\mathrm{Z}}+S_{1}^{\mathrm{Z}}=0 ; \quad \text { for } T>T_{\mathrm{C}} .
$$

Specially for DTGS crystal (ferroelectric), both spins become zero.

$$
\begin{array}{cl}
S_{1}^{\mathrm{Z}}=S_{1}^{\mathrm{Z}}=0 ; & \begin{array}{l}
\text { for DTGS crystal (ferroelectric) at } \\
\text { phase transition, }
\end{array}
\end{array}
$$


while $S_{1}^{\mathrm{Z}} ; S_{1}^{\mathrm{Z}}>0$; but $P_{\mathrm{S}} \rightarrow 0$ not exactly zero (for second order phase transition)

$$
\begin{aligned}
& <S^{x}>=\frac{\Omega}{\tilde{\Omega}} \tan h\left(\frac{\beta \tilde{\Omega}}{2}\right) \\
& <S^{y}>=0 \\
& <S^{z}>=\frac{\left(J_{0}<S^{Z}>+J_{0}^{\prime}<S^{Z}>^{3}\right)}{2 \tilde{\Omega}} \tan h\left(\frac{\beta \tilde{\Omega}}{2}\right),
\end{aligned}
$$

Having differentiated twice the Green's function (2) first with respect to time $(t)$ and then with respect to time $\left(t^{\prime}\right)$ using model Hamiltonian (1) taking Fourier transformation and setting it into Dyson's equation from

$$
G_{i j}(\omega)=G_{i j}^{0}(\omega)+G_{i j}^{0}(\omega) P(\omega) G_{i j}^{0}(\omega),
$$

where

$$
\begin{aligned}
G_{i j}^{0}(\omega) & =\frac{\Omega\left\langle S_{1 i}^{x}\right\rangle \delta_{i j}}{\pi\left(\omega^{2}-4 \Omega^{2}\right)}, \\
G_{i j}(\omega) & =\frac{\Omega\left\langle S_{1 i}^{x}\right\rangle}{\pi\left(\omega^{2}-4 \Omega^{2}-P(\omega)\right)},
\end{aligned}
$$

where

$$
\begin{aligned}
& P(\omega)=\tilde{P}(\omega)+\tilde{\tilde{P}}(\omega), \\
& \tilde{P}(\omega)=\frac{\pi i}{\Omega\left\langle S_{1 i}^{x}\right\rangle^{2}}\left\langle F_{i}^{(t)} ; S_{i j}^{y}\right\rangle,
\end{aligned}
$$

and,

$$
\tilde{\tilde{P}}(\omega)=\frac{\pi^{2}}{\Omega^{2}\left\langle S_{1 i}^{x}\right\rangle^{2}}\left\langle\left\langle F_{i}, F_{j}\right\rangle\right\rangle .
$$

The second term of (6) contains higher order Green's functions which are decoupled by using scheme $<$ abcd $>=$ $<a b><c d>+<a c><b d>+<a d><b c>$. Then simpler Green's functions are solved in the zeroth order approximation, i.e. higher order terms are neglected from $P(\omega)$ type terms. In $(8), \tilde{\tilde{P}}(\omega)$ is resolved into its real and imaginary parts using formula

$$
\lim _{m \rightarrow 0} \frac{1}{x+\mathrm{im}}=\left(\frac{1}{x}\right) \pm i \pi \delta(x) .
$$

The real part is known as shift $\Delta(\omega)$ and the imaginary part is called width $\Gamma(\omega)$.

These are obtained as spin shift;

$$
\begin{aligned}
\Delta_{s}(\omega)= & \frac{a^{4}}{2 \Omega\left(\omega^{2}-\tilde{\Omega}^{2}\right)}+\frac{b^{2} c^{2}}{4 \Omega \tilde{\Omega}}+\frac{V_{i k}^{2} N_{k} a^{2}}{2 \Omega\left(\omega^{2}-\tilde{\Omega}^{2}\right)} \\
& +\frac{4 \mu^{2} E^{2} a^{2}}{2 \Omega\left(\omega^{2}-\tilde{\Omega}^{2}\right)}
\end{aligned}
$$

$$
\Delta_{s-p}(\omega)=\frac{2 V_{i k}^{2}\left\langle S_{1 i}^{x}\right\rangle \omega_{k} \delta_{k k^{i}}\left(\omega^{2}-\tilde{\tilde{\omega}}_{k}^{2}\right)}{\left[\left(\omega^{2}-\tilde{\tilde{\Omega}}_{k}^{2}\right)^{2}+4 \omega_{k}^{2} \Gamma_{k}^{2}(\omega)\right]},
$$

spin width is

$$
\begin{aligned}
\Gamma(\omega)= & \frac{\pi a^{4}}{4 \Omega \tilde{\Omega}}[\delta(\omega-\tilde{\Omega})-\delta(\omega+\tilde{\Omega})] \\
& +\frac{b^{2} c^{2}}{4 \Omega \tilde{\Omega}}[\delta(\omega-\tilde{\Omega})-(\omega+\tilde{\Omega})] \\
& +\frac{V_{i k}^{2} N_{k} a^{2}}{4 \Omega \tilde{\Omega}}[\delta(\omega-\tilde{\Omega})-\delta(\omega+\tilde{\Omega})] \\
& +\frac{2 \pi \mu^{2} E^{2} a^{2}}{4 \Omega \tilde{\Omega}}[\delta(\omega-\tilde{\Omega})-\delta(\omega+\tilde{\Omega})],
\end{aligned}
$$

spin phonon width is

$$
\Gamma_{s-p}(\omega)=\frac{4 V_{i k}^{2}\left\langle S_{1 i}^{x}\right\rangle \omega_{k}\left(\omega^{2}-\tilde{\tilde{\omega}}_{k}^{2}\right)}{\left[\left(\omega^{2}-\tilde{\tilde{\omega}}_{k}^{2}\right)^{2}+4 \omega_{k}^{2} \Gamma_{k}^{2}(\omega)\right]} .
$$

In (10) and (12), $\tilde{\tilde{\omega}}_{k}$ is renormalized phonon frequency and $\gamma_{k}(\omega)$ the phonon width in the Green's function $G_{k k^{\prime}}(t-$ $\left.t^{\prime}\right)=<<A_{k}(t) ; A_{k^{1}}\left(t^{\prime}\right)>>$ which are obtained as

$$
G_{i j^{\prime}}(\omega)=\frac{\omega_{k} \delta_{k k^{\prime}}}{\pi\left[\omega^{2}-\tilde{\tilde{\omega}}_{k}^{2}-2 \omega_{k}\left\{\Delta_{k}(\omega)+i \Gamma(\omega)\right]\right.},
$$

$$
\tilde{\tilde{\omega}}_{k}^{2}=\tilde{\omega}_{k}^{2}+2 \omega_{k} \Delta_{k}(\omega)
$$

phonon shift is given as

$$
\begin{aligned}
& \Delta_{k}(\omega)=\operatorname{Re} P_{k}(\omega) \\
& =18 P \sum_{k_{1} k 2}\left|V^{(3)}\left(k_{1}, k_{2},-k\right)\right|^{2} \text {, } \\
& \frac{\omega_{k 1} \omega_{k 2}}{\tilde{\omega}_{k 1} \tilde{\omega}_{k 2}}\left\{\left(n_{k 1}+n_{k 2}\right) \frac{\tilde{\omega}_{k 1}+\tilde{\omega}_{k 2}}{\omega^{2}-\left(\tilde{\omega}_{k 1}+\tilde{\omega}_{k 2}\right)^{2}}\right. \\
& \left.+\left(n_{k_{2}}-n_{k_{1}}\right) \frac{\tilde{\omega}_{k 1}+\tilde{\omega}_{k 2}}{\omega^{2}-\left(\tilde{\omega}_{k 1}+\tilde{\omega}_{k 2}\right)^{2}}\right\} \\
& +48 P \sum_{k 1_{1} k_{2 k_{3}}}\left|V^{(4)}\left(k_{1} k_{2}, k_{3},-k\right)\right|^{2} \frac{\omega_{k_{1}} \omega_{k 2} \omega_{k 3}}{\tilde{\omega}_{k_{1}} \tilde{\omega}_{k_{2}} \tilde{\omega}_{k 3}} \\
& \left\{\left(1+n_{k_{1}} n_{k_{2}}+n_{k_{2}} n_{k_{3}}+n_{k_{3}} n_{k_{1}}\right)\right. \\
& \times \frac{\tilde{\omega}_{k_{1}}+\tilde{\omega}_{k 2}+\tilde{\omega}_{k 3}}{\omega^{2}-\left(\tilde{\omega}_{k_{1}}+\tilde{\omega}_{k_{2}}+\tilde{\omega}_{k 3}\right)^{2}} \\
& +3\left(1-n_{k_{2}} n_{k_{1}}+n_{k 2} n_{k_{3}}-n_{k_{3}} n_{k_{1}}\right) \\
& \left.\frac{\tilde{\omega}_{k_{1}}+\tilde{\omega}_{k 2}+\tilde{\omega}_{k 3}}{\omega^{2}-\left(\tilde{\omega}_{k_{1}}+\tilde{\omega}_{k_{2}}+\tilde{\omega}_{k 3}\right)^{2}}\right\} \text {. }
\end{aligned}
$$


and phonon width is given as

$$
\begin{aligned}
\Gamma_{k}(\omega)= & \operatorname{Im} P_{k}(\omega) \\
= & 9 \pi \sum_{k_{1} k_{2}}\left|V^{(3)}\left(k_{1}, k_{2},-k\right)\right|^{2} \frac{\omega_{k_{1}} \omega_{k_{2}}}{\tilde{\omega}_{k_{1}} \tilde{\omega}_{k_{2}}} \\
& \left\{\left(n_{k_{1}}+n_{k_{2}}\right)\left[\delta\left(\omega+\tilde{\omega}_{k_{1}}+\tilde{\omega}_{k_{1}}\right)\right]\right. \\
& \quad-\delta\left(\omega-\tilde{\omega}_{k_{1}}-\tilde{\omega}_{k_{1}}\right)+(\text { higher terms }) \\
& +\left(n_{k_{2}}-n_{k_{1}}\right)\left[\delta\left(\omega+\tilde{\omega}_{k_{1}}+\tilde{\omega}_{k_{2}}\right)\right. \\
& \left.\left.\quad \delta\left(\omega+\tilde{\omega}_{k_{1}}+\tilde{\omega}_{k_{2}}\right)\right]\right\} \\
+ & \left.48 \pi \sum^{(4)}\left(k_{1}, k_{2}, k_{3},-k_{4}\right)\right|^{2} \frac{\omega_{k_{1}} \omega_{k_{2}} \omega_{k_{3}}}{\tilde{\omega}_{k_{1}} \tilde{\omega}_{k_{2}} \tilde{\omega}_{k_{3}}} \\
& \mathrm{X}\left\{\left(1+n_{k_{1}} n_{k_{2}}+n_{k_{2}} n_{k_{3}}+n_{k_{3}} n_{k_{4}}\right)\right. \\
& X\left[\delta\left(\omega+\tilde{\omega}_{k_{1}}+\tilde{\omega}_{k_{2}}+\tilde{\omega}_{k_{3}}\right)\right. \\
& \left.\quad-\delta\left(\omega-\tilde{\omega}_{k_{1}}-\tilde{\omega}_{k_{2}}-\tilde{\omega}_{k_{3}}\right)\right] \\
+ & 3\left(n_{k_{1}} n_{k_{2}}+n_{k_{2}} n_{k_{3}}-n_{k_{3}} n_{k_{4}}\right) \\
+ & 3\left(n_{k_{1}} n_{k_{2}}+n_{k_{2}} n_{k_{3}}-n_{k_{3}} n_{k_{4}}\right) \\
X & \quad \delta\left(\omega+\tilde{\omega}_{k_{1}}-\tilde{\omega}_{k_{2}}-\tilde{\omega}_{k_{3}}\right) \\
& \left.\left.\quad-\delta\left(\omega-\tilde{\omega}_{k_{1}}+\tilde{\omega}_{k_{2}}+\tilde{\omega}_{k_{3}}\right)\right]\right\} \\
& \quad(14 \mathrm{c}) \\
\tilde{\omega}_{k}^{2}=\tilde{\omega}_{k}^{2}+A_{k}(T), &
\end{aligned}
$$

The Green's function (2) finally becomes

$$
\begin{aligned}
& G_{i j}(\omega)=\frac{\Omega\left\langle S_{1 i}^{x}\right\rangle \delta_{i j}}{\pi\left(\omega^{2}-\hat{\Omega}^{2}-P(\omega)\right)}, \\
& \hat{\Omega}^{2}=\tilde{\tilde{\Omega}}^{2}+2 \Omega \Delta_{s-p}(\omega), \\
& \tilde{\Omega}^{2}=\tilde{\Omega}^{2}+2 \Omega \Delta_{s}(\omega), \\
& \tilde{\Omega}^{2}=4 \Omega^{2}+\frac{1}{\Omega\left\langle S_{1 i}^{x}\right\rangle}\left\langle\left[F, S_{1 j}^{y}\right]\right\rangle,
\end{aligned}
$$

In (18), second term is evaluated using mean field approximation, i.e.

$$
\frac{\left\langle S_{1 i}^{z}\right\rangle}{a}=\frac{\left\langle S_{1 i}^{x}\right\rangle}{b}=\frac{1}{2 \tilde{\Omega}} \tanh \beta \frac{\tilde{\Omega}}{2},
$$

which gives

$$
\tilde{\Omega}^{2}=a^{2}+b^{2}+b c,
$$

where

$$
\begin{aligned}
& a=2 J\left\langle S_{1}^{z}\right\rangle+K\left\langle S_{2}^{z}\right\rangle+2 \mu E, \\
& b=2 \Omega ;
\end{aligned}
$$

and

$$
c=2 J\left\langle S_{1}^{x}\right\rangle+K\left\langle S_{2}^{x}\right\rangle,
$$

solving (16)

$$
\begin{aligned}
& \begin{aligned}
\hat{\Omega}_{ \pm}^{2}=\frac{1}{2}\left(\tilde{\tilde{\omega}}_{k}^{2}+\tilde{\tilde{\Omega}}^{2}\right) \pm \frac{1}{2}[ & \left(\tilde{\tilde{\omega}}_{k}^{2}-\tilde{\tilde{\Omega}}^{2}\right)^{2} \\
& \left.+8 V_{i k}^{2}\left\langle S_{1 i}^{x}\right\rangle \Omega \omega_{k}\right]^{1 / 2}
\end{aligned} \\
& T_{c}=\frac{\eta}{2 k_{B} \tan h^{-1}\left(\frac{\eta^{3}}{4 \Omega^{2} J^{*}}\right)}, \\
& \eta^{2}=(2 J-K)^{2} \sigma^{2}+4 \Omega^{2}+(2 \mu E)^{2}
\end{aligned}
$$

where

and

$$
(J+K)^{*}=(2 J+K)+\frac{2 V_{i k}^{2} \tilde{\tilde{\omega}}_{k}^{2}}{\left[\tilde{\tilde{\omega}}_{k}^{4}+4 \omega_{k} \Gamma_{k}^{2}\right]} .
$$

$J^{*}$ is renormalized exchange interaction constant.

\subsection{Dielectric constant and loss tangent}

The response of a dielectric crystal to the external electric field is expressed as dielectric susceptibility, $\chi$, given by

$$
\chi(\omega)=-\lim _{X \rightarrow 0} 2 \pi N \mu^{2} G_{i j}(\omega+i X) .
$$

The $\chi(\omega)$ is related to dielectric constant as

$$
\varepsilon=1+4 \pi \chi
$$

With the help of (25) and (26), one obtains expression for dielectric constant as

$$
\varepsilon(\omega)=\left(-8 \pi N \mu^{2}\right) \frac{\left\langle S_{1}^{x}\right\rangle \Omega}{\left[\left(\omega^{2}-\hat{\Omega}^{2}\right)^{2}+4 \Omega^{2} \Gamma^{2}\right]},
$$

$\varepsilon(\omega)>>1$ in the ferroelectric crystal. The power lost in dielectric when exposed to electromagnetic field is conveniently shown as dielectric tangent loss which is expressed as

$$
\tan \delta=\frac{\varepsilon^{\prime \prime}}{\varepsilon^{\prime}},
$$

By using (30) and (31), we obtain expression for loss tangent as

$$
\tan \delta=-\frac{2 \Omega \Gamma(\omega)}{\left(\omega^{2}-\hat{\Omega}^{2}\right)} .
$$

\section{4 - -factor}

$$
Q \text {-factor }=1 / \tan \delta,
$$

where, $\tan \delta=$ tangent loss. 


\subsection{Acoustic attenuation}

The acoustic attenuation is given as

$$
\alpha=\frac{\Gamma(\omega)}{v}
$$

where $\Gamma(\omega)$ is width and $v$ the sound velocity.

\subsection{Ratio of figure of merits}

We know that dielectric permittivity can be written as

$$
\varepsilon=\varepsilon^{\prime} \pm i \varepsilon^{\prime \prime}
$$

where $\varepsilon^{\prime}$ is the real part of permittivity, $\varepsilon^{\prime \prime}$ the imaginary part of permittivity, and magnitude of this complex number will be

$$
\left.\varepsilon={\sqrt{\left(\varepsilon^{\prime}\right.}}^{2}+\varepsilon^{\prime \prime 2}\right)
$$

And dielectric loss

$$
\tan \delta=\frac{\varepsilon^{\prime \prime}}{\varepsilon^{\prime}} .
$$

Solving, (35) and (36), we have

$$
\varepsilon^{\prime}=\sqrt{\left(\frac{\varepsilon^{2}}{1+\tan ^{2} \delta}\right)}
$$

and

$$
\varepsilon^{\prime \prime}=\sqrt{\frac{\varepsilon^{2} \tan ^{2} \delta}{1+\tan ^{2} \delta}} .
$$

In IR detection, the figure of merit can be written as

$$
M_{1}=\frac{\rho}{\varepsilon^{\prime}} .
$$

High current reponsivity

$$
M_{2}=\frac{\rho}{\sqrt{\varepsilon^{\prime \prime}}}
$$

And vidicons application index

$$
M_{3}=\frac{\rho}{\sqrt{\varepsilon^{\prime}}} .
$$

Then relative figure of merits will be

$$
\begin{aligned}
& \frac{M_{2}}{M_{1}}=\sqrt{\varepsilon^{\prime \prime}}, \\
& \frac{M_{3}}{M_{2}}=\sqrt{\frac{\varepsilon^{\prime \prime}}{\varepsilon^{\prime}}}, \\
& \frac{M_{3}}{M_{1}}=\frac{\varepsilon^{\prime \prime}}{\sqrt{\varepsilon^{\prime}}} .
\end{aligned}
$$

\subsection{Electric conductivity}

$$
\sigma=\omega \varepsilon_{0} \varepsilon^{\prime \prime}
$$

where $\sigma$ is the electric conductivity and $\omega$ the phonon frequency.

\subsection{Relaxation time (minimum)}

Relaxation time (minimum) is,

$$
\tau=\frac{\exp ^{-\left(\frac{\pi}{2} \tan \delta\right)}}{\omega} .
$$

\subsection{Differentiability}

Smooth function $=\frac{2 \varepsilon^{\prime \prime}}{\pi \varepsilon}$.

\section{Numerical calculation and results}

By using model values of various parameters in expression from literature (table 1), temperature and electric field dependence of soft mode frequency dielectric constant, tangent loss, quality factor, acoustic attenuation, electric conductivity, smooth function, relaxation time ( $\mathrm{min}$ ) and ratio of figure of merits of deuterated triglycine sulphate crystal have been calculated and shown in figures 2-14.

\section{Discussion}

Here we have discussed a theory, which is actually a combination of three different theories i.e. pseudo spin lattice coupled mode (PLCM) model, Ising spin model and Green's function theory, in which lattice dynamics of crystal decide the shape of modelling for specific deuterated triglycine sulphate crystal $\left(\mathrm{CD}_{2} \mathrm{ND}_{2} \mathrm{COOD}\right)_{3} \mathrm{D}_{2} \mathrm{SO}_{4}$. We have extended

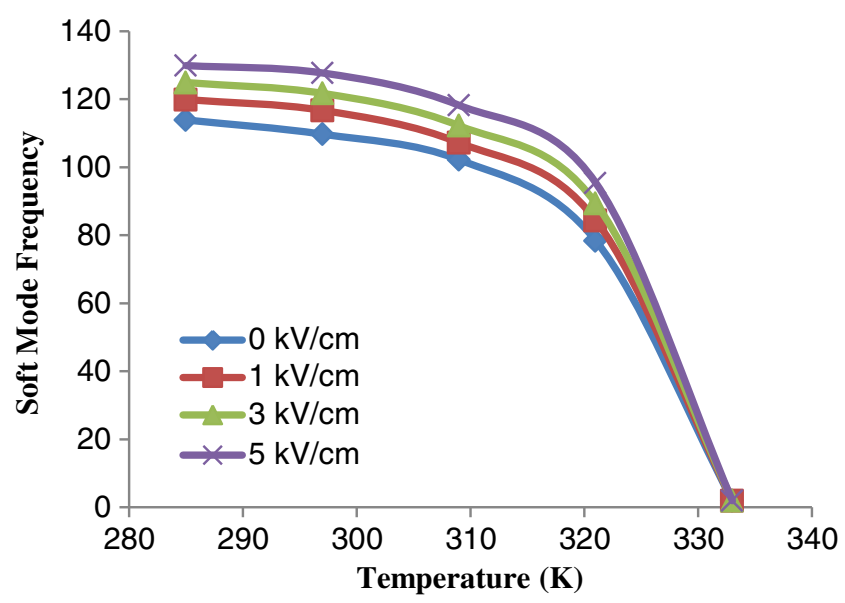

Figure 2. Temperature and electric field dependent soft-mode frequency of deuterated triglycine sulphate crystal (experimentally proved). 


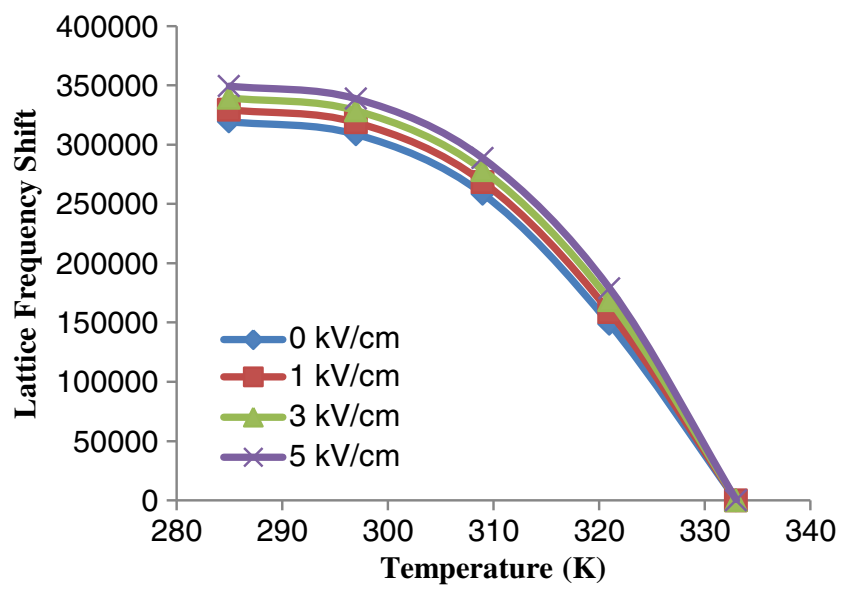

Figure 3. Temperature and electric field dependent soft-mode frequency of deuterated triglycine sulphate crystal (experimentally proved).

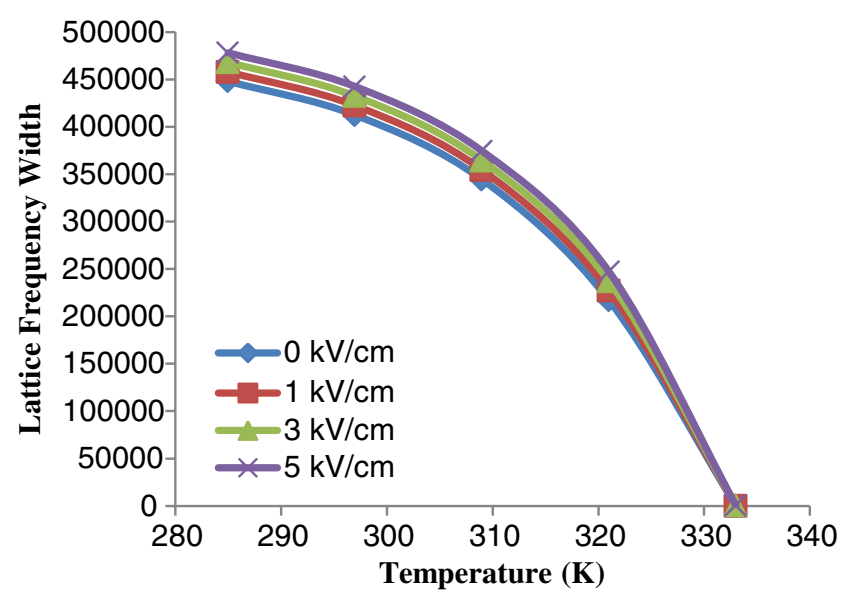

Figure 4. Temperature and electric field dependent soft-mode frequency of deuterated triglycine sulphate crystal (experimentally proved).

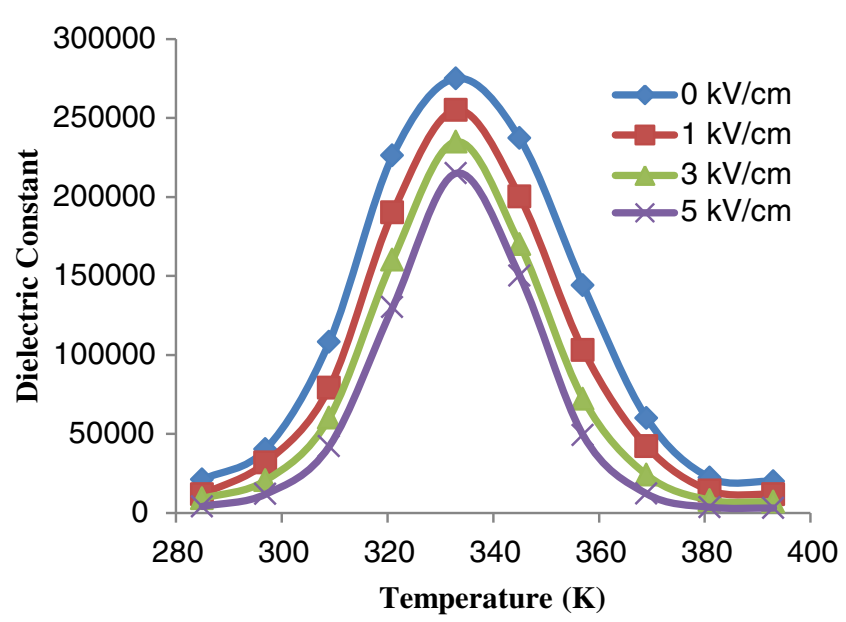

Figure 5. Temperature and electric field dependent dielectric constant of deuterated triglycine sulphate crystal (experimentally matched with Beerman 1974).

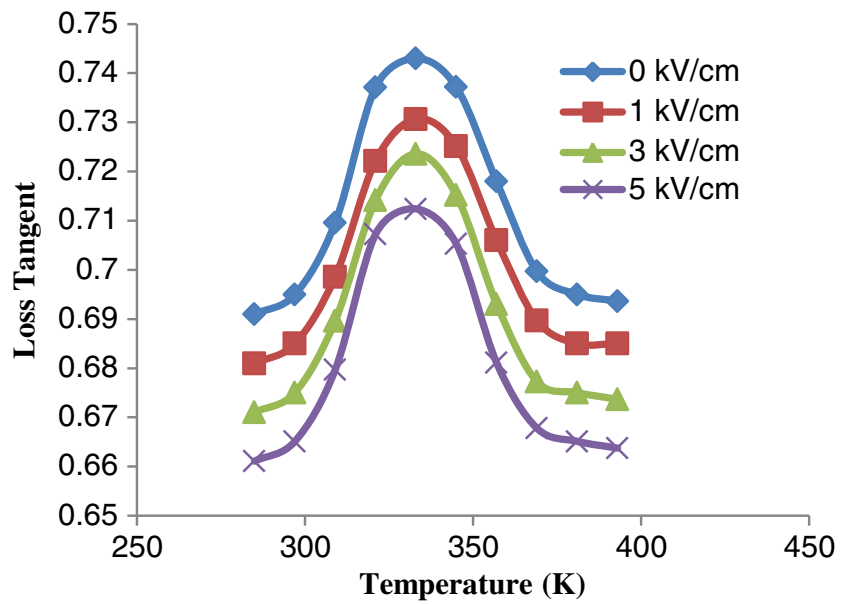

Figure 6. Temperature and electric field dependent loss tangent of deuterated triglycine sulphate crystal (experimentally matched with Beerman 1974).

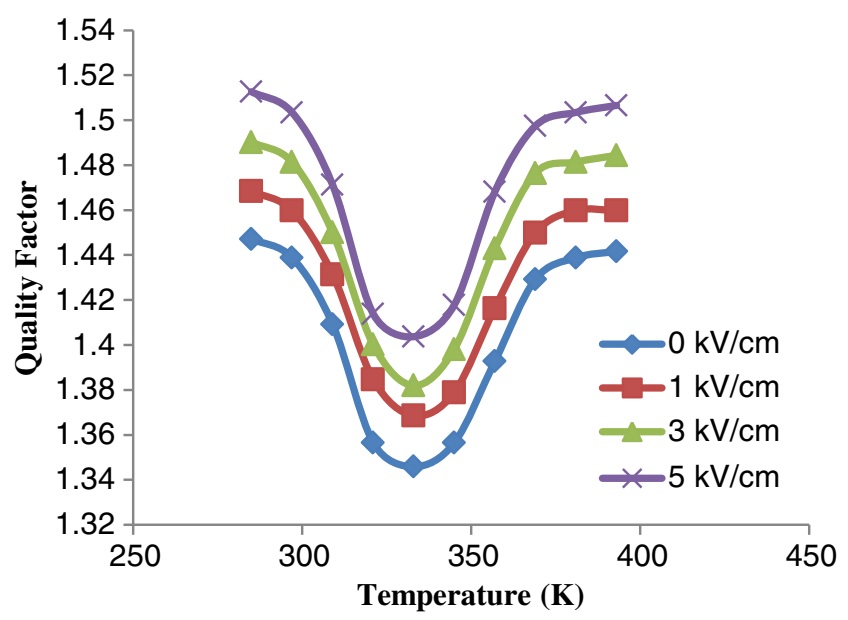

Figure 7. Temperature and electric field dependent quality factor of deuterated triglycine sulphate crystal (experimentally matched with Beerman 1974).

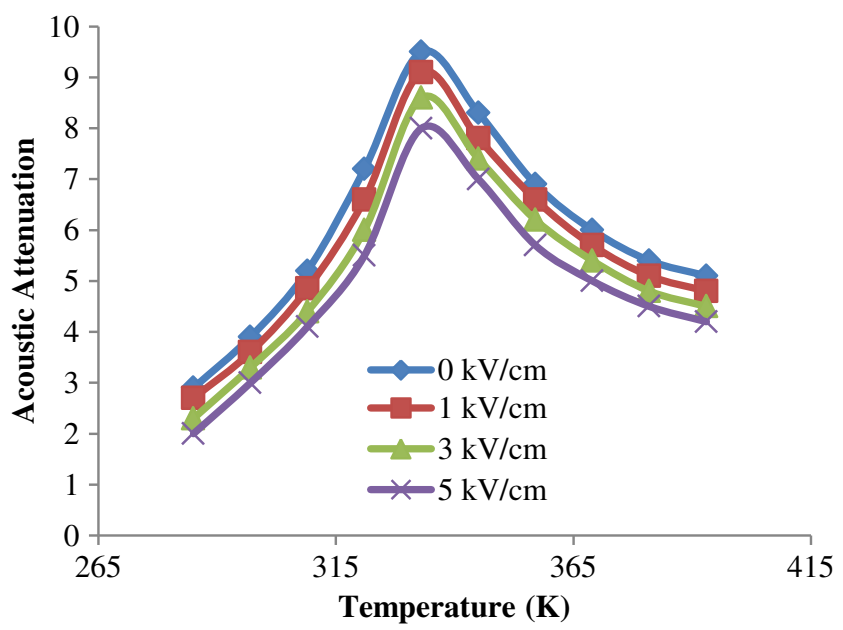

Figure 8. Temperature and electric field dependent acoustic attenuation of deuterated triglycine sulphate crystal (experimentally proved). 


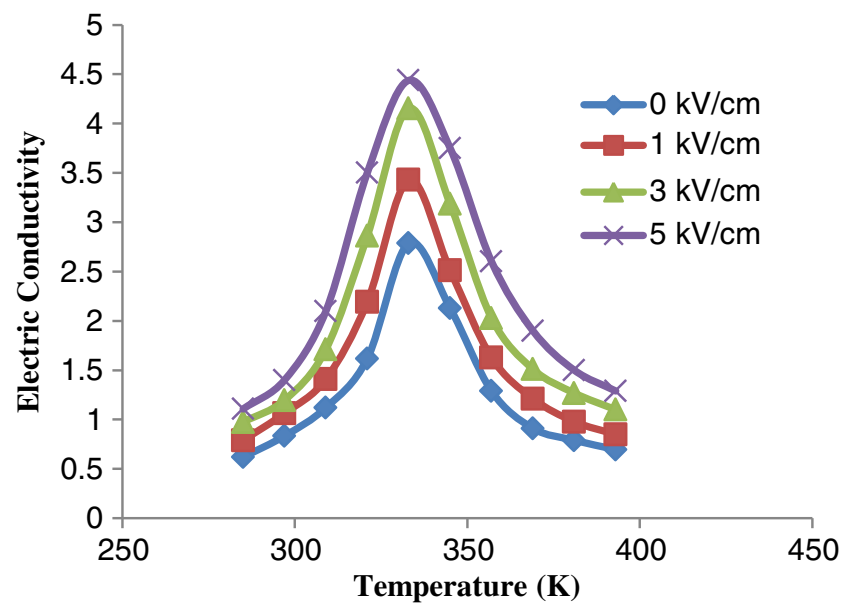

Figure 9. Temperature and electric field dependent electric conductivity of deuterated triglycine sulphate crystal (experimentally proved).

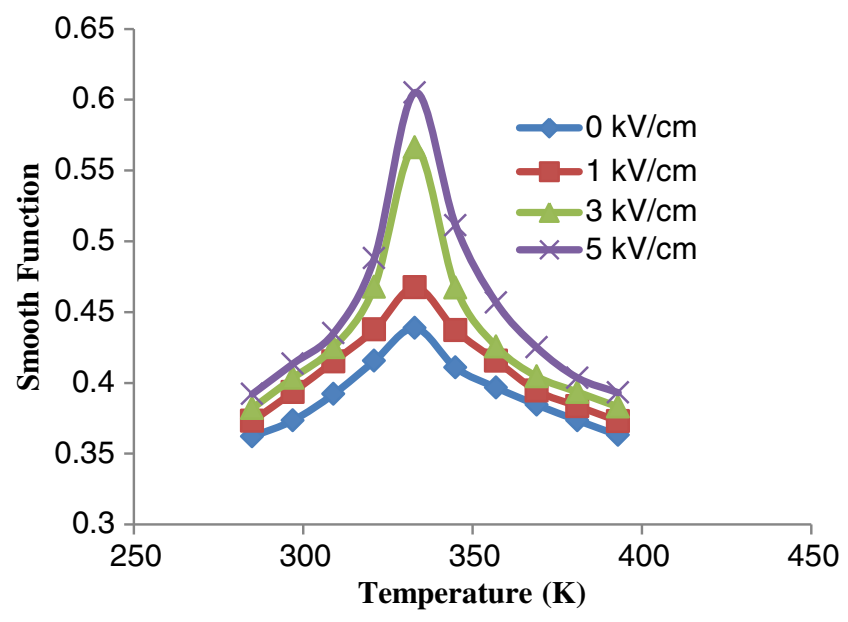

Figure 10. Temperature and electric field dependent smooth function of deuterated triglycine sulphate crystal (experimentally proved).

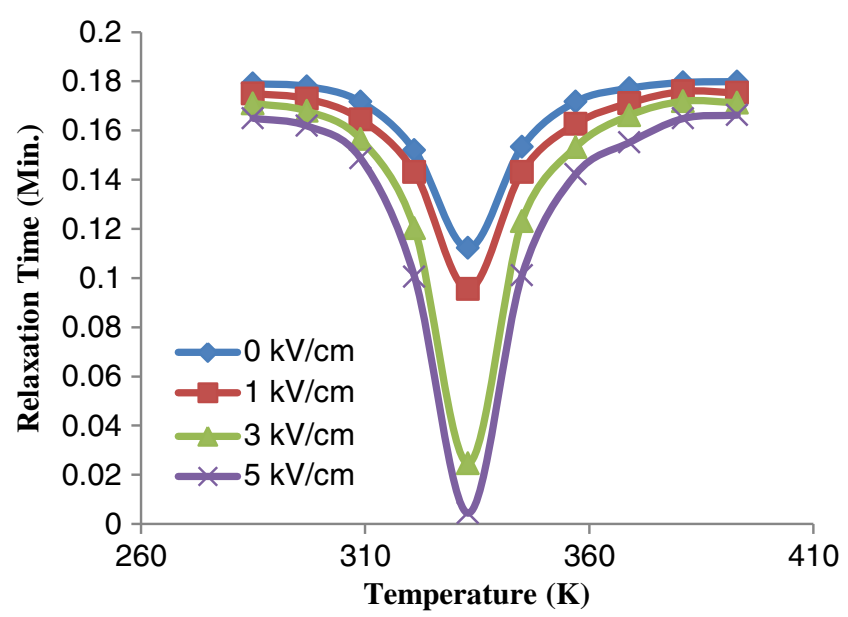

Figure 11. Temperature and electric field dependent relaxation time ( $\mathrm{min}$ ) of deuterated triglycine sulphate crystal (experimentally matched with Drozhdin et al 2006).

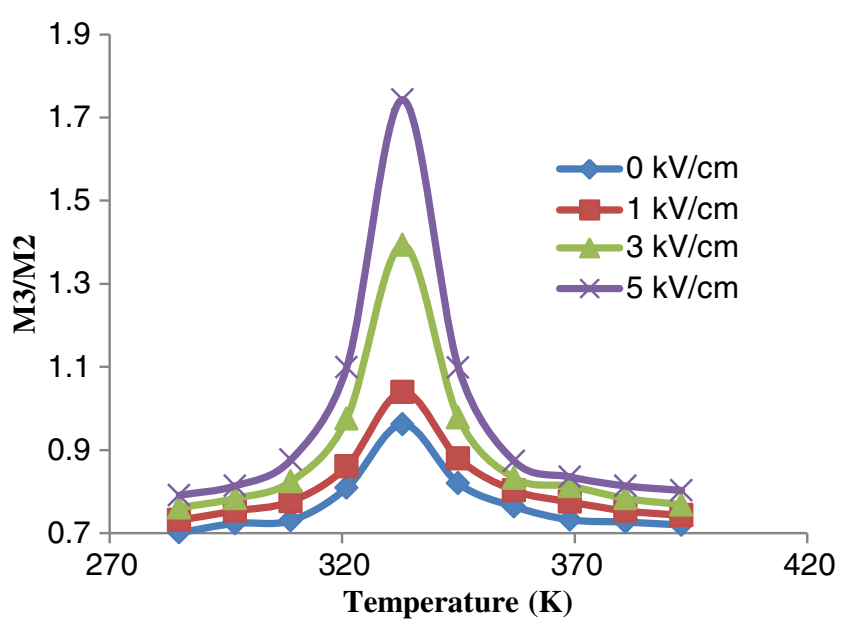

Figure 12. Temperature and electric field dependent ratio of figure of merits $(M 3 / M 2)$ of deuterated triglycine sulphate crystal (experimentally matched with Batra et al 2003).

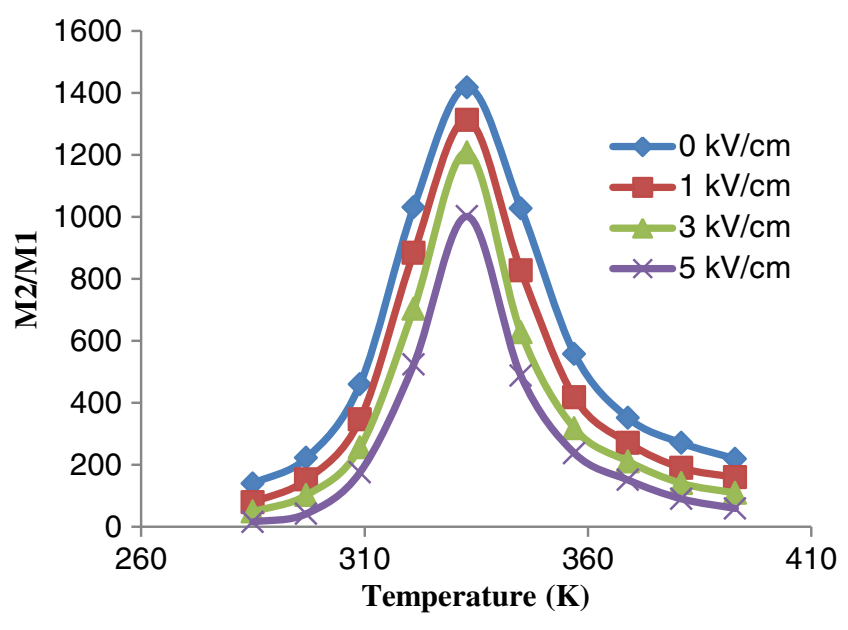

Figure 13. Temperature and electric field dependent ratio of figure of merits $(M 2 / M 1)$ of deuterated triglycine sulphate crystal (experimentally matched with Batraet al 2003).

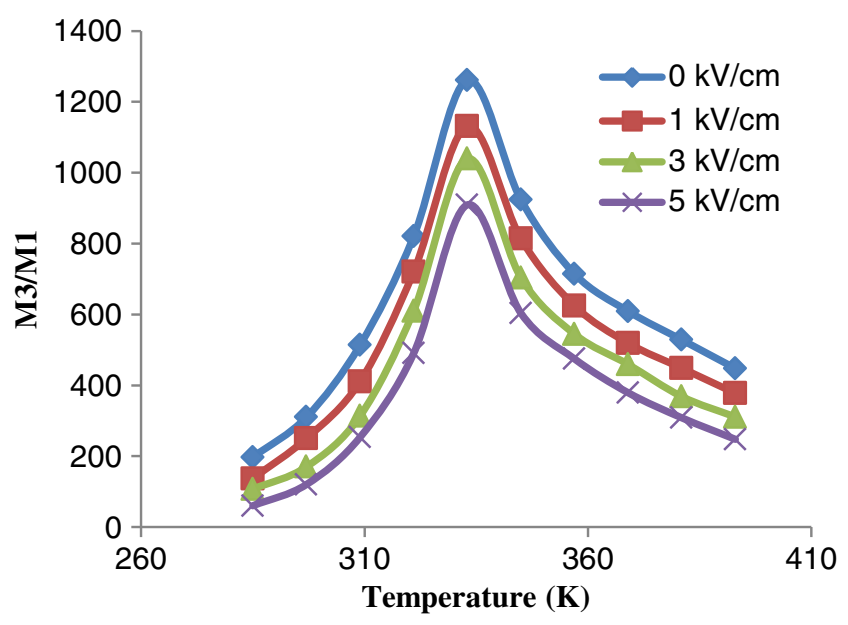

Figure 14. Temperature and electric field dependent ratio of figure of merits $(M 3 / M 1)$ of deuterated triglycine sulphate crystal (experimentally matched with Batra et al 2003). 
the present PLCM model with anharmonic vibration of the lattice till fifth order which gives much accurate Hamiltonian of the crystal also in electric field condition. We have calculated soft-mode frequency in each crystal which is nearly to zero $\left(\approx 10^{-1}\right.$ order $)$ at respective Curie's temperature. It increases when we apply higher order of fields on crystal. Dielectric permittivity of each crystal is very high $(\approx$ $10^{4}-10^{5}$ order) at Curie's temperature and decreases with higher order of fields. The loss can be explained as follows: "A transverse radiation field derives the low lying transverse mode of the material in a forced vibration. Energy is transferred from the electromagnetic field to this lattice mode and is then degraded into other vibrational modes of the material. Due to anharmonic phonon interactions, decay processes take place, for example, third order interaction leads to the decay of a virtual phonon in the real phonons or the virtual phonons may be destroyed by scattering a thermal excited phonon." Tangent loss has maximum value less than unity $(\approx$ $10^{-1}$ order) at phase transition and it decreases with higher order of fields while quality factor is reciprocal of tangent loss which is minimum at Curie's temperature and increases with high order electric field. Acoustic attenuation of the crystal has been calculated. This is maximum $\left(\approx 10^{0}-10^{1}\right.$ order) at Curie's temperature and decreases with higher order of fields. We have also observed that, in electric biased condition, Curie's temperature slightly displaced (decreased) from it's value. Our calculated data fully agreed with experimental results.

\section{Conclusions}

The present study proposes much reliable and authentic data about hydrogen bonded deuterated triglycine sulphate crystal properties in soft mode vibrations as well as acoustic mode vibrations. It considers higher order interaction between two lattices, harmonic as well as anharmonic interactions even with electric field conditions. Theoretical results are fairly in agreement with experimental data (Beerman 1974; Drozhdin et al 2003; Batra et al 2003), so that we can conclude that the present model is applicable to all hydrogen bonded pyroelectric ferroelectric crystals (triglycine group crystals mostly).

\section{Acknowledgements}

The authors are thankful to Prof. P K Bajpai, Guru Ghasidas University, Chhattisgarh, Prof. K K Verma, RML Avadh University, Prof. Shyam Kumar, Kurukshetra University and Prof. Shafikuddin Mollah, A M University, Aligarh, for their kind suggestions and Dr R P Pant, NPL, Delhi, Prof S C Bhatt (HNBGU), Prof. R P Gairola, HNBGU, Srinagar, Prof. U C Nathani, HNBGU Pauri, Dr K S Bartwal, RRCAT, Indore, Dr Sudhir Kumar, Rohilkhand University, Bareilly, Dr Vinay Gupta, Delhi University and Dr S K Srivastava, Bundelkhand University, Jhansi, for their kind encouragement.

\section{References}

Batra A K, Aggarwal M D and Lal R B 2003 Mater. Letts 573943

Beerman H P 1974 Ferroelectrics 8653

Bjorkstam J L 1967 Phys. Rev. 153599

Blinc R, Detoni S and Pintar M 1961 Phys. Rev. 1241036

Brezina B and Smutny F 1968 Czech. J. Phys. 18393

Camnasio A and Gonzalo J A 1975 Solid State Commun. 161169

Chaudhuri B K, Chaudhari K R and Banerjee S 1988 Phys. Rev. B38 689

Drozhdin S N, Golitsyna O M, Nikishina A I, Tuma F A and Tarasov D P 2006 Phys. Solid State 48532

Gonzalo J A 1970 Phys. Rev. B1 3125

Kushnir O S 2003 Phys. Status Solidi 236191

Mathias B T, Miller C E and Remeika J P 1956 Phys. Rev. 104849

Mitsui T 1958 Phys. Rev. 1111259

Tello M J and Hernandez E 1973 J. Phys. Soc. Jpn 351289 